PROCEEDINGS OF THE

AMERICAN MATHEMATICAL SOCIETY

Volume 124, Number 12, December 1996, Pages 3673-3676

S 0002-9939(96)03922-6

\title{
AN EXTENSION \\ OF THE VITALI-HAHN-SAKS THEOREM
}

\author{
ONÉSIMO HERNÁNDEZ-LERMA AND JEAN B. LASSERRE \\ (Communicated by Christopher D. Sogge)
}

\begin{abstract}
The Vitali-Hahn-Saks theorem on the absolute continuity of the setwise limit of a sequence of bounded measures is extended to allow unbounded measures and convergence of integrals of continuous functions vanishing at infinity.
\end{abstract}

\section{INTRODUCTION}

A useful version of the Vitali-Hahn-Saks theorem gives conditions for a measure $\lambda$ which is the setwise limit of a sequence of absolutely continuous (a.c.) measures $\left\{\lambda_{n}\right\}$ to be a.c. This result allows the underlying measure space $(X, \mathcal{B})$ to be arbitrary (cf. [2], p. 155), but it requires that $\left\{\lambda_{n}\right\}$ and $\lambda$, as well as the dominant measure, say $\mu$, are all finite.

In this paper, we show that the same conclusion $(\lambda \ll \mu)$ holds without requiring either setwise convergence or finiteness of the measures involved, but we impose in particular suitable topological conditions on $X$.

Moreover, we give conditions for the Radon-Nikodym derivative $d \lambda / d \mu$ to be in $L_{p}(X, \mathcal{B}, \mu)$ for $1 \leq p \leq \infty$.

In addition to being interesting in themselves, these results are also useful to study the existence of solutions to the Poisson Equation for a Markov kernel [3].

\section{MAIN RESUlts}

Let $(X, \mathcal{B}, \mu)$ be a $\sigma$-finite complete measure space, where $X$ is a locally compact separable metric space, and $\mathcal{B}$ is the completion (with respect to $\mu$ ) of the $\sigma$-algebra of Borel subsets of $X$. Let $C_{0}(X)$ be the space of real-valued continuous functions on $X$ vanishing at infinity. (Concerning the convergence in (2.1), see Remark 2.3(b) at the end of this section.)

Received by the editors January 24, 1995.

1991 Mathematics Subject Classification. Primary 28A33; Secondary 28C15.

Key words and phrases. Measures, setwise convergence, absolute continuity.

The research of the first author was partially supported by a visiting professorship at Paul Sabatier University, Toulouse, France, and by CONACYT Grant 1332-E9206.

This research was partially supported by the CNRS (France)- CONACYT (México) Scientific Cooperation Program.

(C)1996 American Mathematical Society 
Theorem 2.1. Let $\left\{\lambda_{n}\right\}$ and $\lambda$ be $\sigma$-finite (nonnegative) measures on $(X, \mathcal{B})$ such that, as $n \rightarrow \infty$,

$$
\int_{X} v d \lambda_{n} \rightarrow \int_{X} v d \lambda \forall v \in C_{0}(X) .
$$

If, in addition, every $\lambda_{n}$ is a.c. with respect to $\mu$, then so is $\lambda$.

Suppose that the measures $\lambda$ and $\lambda_{n}$ in Theorem 2.1 are finite. Then, by the Radon-Nikodym theorem, there are nonnegative measurable functions $u_{n}$ and $u$ such that

$$
\lambda_{n}(B)=\int_{B} u_{n} d \mu \text { and } \lambda(B)=\int_{B} u d \mu \forall B \in \mathcal{B} .
$$

The next theorem gives conditions for $u$ to be in $L_{p}:=L_{p}(X, \mathcal{B}, \mu), 1 \leq p \leq \infty$.

Theorem 2.2. Fix $1 \leq p \leq \infty$. In addition to the hypotheses of Theorem 2.1, suppose that $u_{n} \in L_{p} \forall n$ and that, for some constant $M$, $\liminf _{n}\left\|u_{n}\right\|_{p} \leq M$. Then

$$
u \text { is in } L_{p} \text {. }
$$

Remark 2.3. (a) If the $\lambda_{n}$ are all finite, then in Theorem 2.1, instead of (2.1), we only need to assume that $\lim _{n} \int_{X} v d \lambda_{n}$ exists and is finite for all $v \in C_{0}(X)$. Then, from [2], Theorem VIII.8, it follows that the sequence $\left\{\lambda_{n}\right\}$ converges to a measure $\lambda$, as in (2.1).

(b) Endowed with the sup-norm, $C_{0}(X)$ is a Banach space and its dual is the space $M(X)$ of finite signed measures on $X$ with total variation norm [2],[5]. Thus, when the $\lambda_{n}$ are finite measures, (2.1) can be stated equivalently as: $\lambda_{n}$ converges to $\lambda$ in the weak* topology $\sigma\left(M(X), C_{0}(X)\right)$. If $\lambda_{n}$ and $\lambda$ are probability measures and (2.1) holds, it is sometimes said that $\lambda_{n}$ converges vaguely to $\lambda$. If $C_{0}(X)$ is replaced by the (larger) space $C(X)$ of all continuous and bounded functions on $X$, it is said that $\lambda_{n}$ converges weakly to $\lambda$ (see e.g. [1]). It follows from Lemma 3.1(b) and the Portmanteau Theorem ([1], Theorem 2.1) that, in this case, vague convergence implies weak convergence (the converse being obviously true).

(c) To verify (2.1) it suffices to consider nonnegative $v \in C_{0}(X)$. Indeed, since

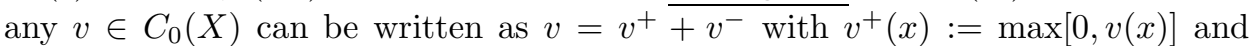
$v^{-}(x):=\min [0, v(x)]$, it suffices to observe that both $v^{+}$and $v^{-}$are in $C_{0}(X)$. Hence, convergence in (2.1) for all $0 \leq v \in C_{0}$ implies convergence for all $v \in C_{0}(X)$.

\section{Proofs}

The proof of Theorem 2.1 is based on the following lemma, which is well known in the case of finite measures (and bounded function $h$ ); see e.g. [2], Theorem VIII.10.

Lemma 3.1. In the context of Theorem 2.1:

(a) If $h$ is a nonnegative and l.s.c. (lower semicontinuous) function on $X$, then

$$
\liminf _{n \rightarrow \infty} \int_{X} h d \lambda_{n} \geq \int_{X} h d \lambda
$$

(b) $\liminf _{n \rightarrow \infty} \lambda_{n}(B) \geq \lambda(B)$ for any open set $B \in \mathcal{B}$. 
Proof. (a) As $h$ is nonnegative and l.s.c., there exists an increasing sequence of nonnegative continuous bounded functions $v_{k}$ on $X$ such that $v_{k}(x) \uparrow h(x) \forall x \in X$. Similarly, as each $v_{k}$ is a nonnegative continuous bounded function and $X$ is $\sigma$ compact ([4], p. 203, Theorem 21), for every $k$ there is an increasing sequence $\left\{v_{k l}, l=1,2, \ldots\right\}$ of nonnegative functions $v_{k l}$ in $C_{0}(X)$ with $v_{k l}(x) \uparrow v_{k}(x)$ for all $x \in X$ as $l \rightarrow \infty$. Hence,

$$
\begin{aligned}
\liminf _{n} \int h d \lambda_{n} & \geq \liminf _{n} \int v_{k} d \lambda_{n} \forall k \\
& \geq \liminf _{n} \int v_{k l} d \lambda_{n} \forall k, l \\
& =\int v_{k l} d \lambda(\text { by }(2.1)) .
\end{aligned}
$$

Thus, letting $l \rightarrow \infty$, and then $k \rightarrow \infty$, the Monotone Convergence Theorem yields (a).

(b) If $B \subset X$ is open, its characteristic (or indicator) function is l.s.c. and of course nonnegative. Hence, (b) follows from (a).

Proof of Theorem 2.1. For every measurable set $B \in \mathcal{B}$ and $\epsilon>0$ there is an open set $G_{\epsilon}$ that contains $B$ and $\mu\left(G_{\epsilon}-B\right)<\epsilon$ (see e.g. [2], p. 41). In particular, if $B$ is a $\mu$-null set, i.e., $\mu(B)=0$ (and hence $\lambda_{n}(B)=0 \forall n$ ), we have $\mu\left(G_{\epsilon}\right)$ $=\mu\left(G_{\epsilon}-B\right)<\epsilon$. Moreover, by part (b) in Lemma 3.1

$$
\liminf _{n} \lambda_{n}\left(G_{\epsilon}\right) \geq \lambda\left(G_{\epsilon}\right) \geq \lambda(B) .
$$

Therefore, letting $\epsilon \downarrow 0$ we obtain $\lambda(B)=0$.

Proof of Theorem 2.2. Consider first the case $p=1$. From $\liminf _{n}\left\|u_{n}\right\|_{1} \leq M$ and Lemma 3.1(b) (with $B:=X$ ), it immediately follows that $\lambda(X)<\infty$ so that $u \in L_{1}$.

Consider now the case $p>1$ and let $q$ be the exponent conjugate to $p$. Then the condition (2.2) holds if $u v$ is in $L_{1}$ for every $v \in L_{q}$ (see e.g. [5], p. 133). In turn, for this to be true it suffices to show that (as $u \geq 0$ ) there is a constant $M$ such that

$$
\int u v d \mu \leq M\|v\|_{q} \forall v \in L_{q}^{+}
$$

Moreover, since (for $1 \leq q<\infty$ ) the class of continuous funtions in $L_{q}$ is dense in $L_{q}$ (see e.g. [2], p. 92) it suffices to prove (3.1) for all continuous functions $v$ in $L_{q}^{+}$. (Indeed, suppose the latter holds, and let $v_{m} \in L_{q}^{+}$be a sequence of continuous functions converging in the $L_{q}$-norm to $v \in L_{q}^{+}$. Let $v_{m_{i}}$ be a subsequence converging to $v \mu$-a.e. Then

$$
\begin{aligned}
M\|v\|_{q}=M \lim _{i}\left\|v_{m_{i}}\right\|_{q} & \geq \lim _{i} \int u v_{m_{i}} d \mu \\
& \geq \int u v d \mu \quad \text { (by Fatou's Lemma); }
\end{aligned}
$$


i.e., $v \in L_{q}^{+}$satisfies (3.1).) Now, let $v \in L_{q}^{+}$be a continuous function. Hence, (2.1) and Lemma 3.1(a) yield:

$$
\begin{aligned}
\int u v d \mu=\int v d \lambda & \leq \liminf _{n} \int v d \lambda_{n} \\
& =\liminf _{n} \int v u_{n} d \mu \\
& \leq \liminf _{n}\left\|u_{n}\right\|_{p}\|v\|_{q} \quad \text { (Hölder's inequality) } \\
& \leq M\|v\|_{q},
\end{aligned}
$$

with $M$ as in Theorem 2.2. As $v$ was an arbitrary continuous function in $L_{q}^{+}$we obtain (3.1), hence (2.2).

\section{REFERENCES}

1. P. Billingsley, Convergence of Probability Measures, Wiley, New York, 1968. MR 38:1718

2. J.L. Doob, Measure Theory, Springer-Verlag, New York, 1994. MR 95c:28001

3. O. Hernández-Lerma and J.B. Lasserre, Existence of solutions to the Poisson equation in $L^{p}$ spaces, LAAS Technical report 95045, Toulouse, 1995.

4. H.L. Royden, Real Analysis, 3rd Edition, Macmillan, New York, 1988. MR 90g:00004

5. W. Rudin, Real and Complex Analysis, 3rd edition, McGraw-Hill, New York, 1986. MR 87f:00009

Departamento de Matemáticas, Cinvestav-ipn, Apdo. Postal 14-740, México D.F. 07000, MeXico

E-mail address: ohernand@math.cinvestav.mx.

LAAS-CNRS, 7 Avenue du Colonel Roche, 31077 Toulouse Cédex, France

E-mail address: lasserre@laas.fr 